Article

\title{
Chip-Based Cytometry Illuminated by a Blade-Shape Continuous Light for Multispectral Detection
}

\author{
Shi-Wei Lin ${ }^{1}$ and Che-Hsin Lin ${ }^{2, *}$ \\ 1 Metal Industries Research \& Development Centre, Kaohsiung 811, Taiwan; strongerlin@mail.mirdc.org.tw \\ 2 Department of Mechanical and Electro-Mechanical Engineering, National Sun Yat-sen University, \\ Kaohsiung 804, Taiwan \\ * Correspondence: chehsin@mail.nsysu.edu.tw; Tel.: +886-9-3284-0730
}

Academic Editors: Teen-Hang Meen and Totaro Imasaka

Received: 9 June 2016; Accepted: 9 August 2016; Published: 13 August 2016

\begin{abstract}
A high performance diascopic illumination configuration is presented for the simultaneous detection of cells and particles with different sizes and different fluorescence labels in a microchannel. In the proposed approach, the cells/particles are illuminated by an objective-type dark-field condenser equipped with a low-cost tungsten light source and are then characterized by extracting the side-scatter, absorbance, and fluorescence signals from the spectra obtained by a ultraviolet-visible-near infrared (UV-Vis-NIR) spectrometer. A modified computation model is adopted to improve the capability for discriminating more fluorescence dyes simultaneously. The feasibility of the proposed detection configuration is demonstrated by counting and classifying a mixed sample of green, red, and crimson fluorescent-labeled particles and non-labeled particles with various dimensions. The suitability of the proposed system for real-world cytometry applications is then evaluated by classifying a mixed bio-sample comprising of gastric epithelial (AGS) cells stained with Trypan-blue and Erythrosin-bluish dye, respectively. The results show that the cytometer enables the efficient detection, identification, and classification of mixed bio-samples without the need for spatial filters or delicate optical components. Consequently, the proposed system has significant potential for high-performance micro-flow cytometry applications.
\end{abstract}

Keywords: micro-flow cytometry; multispectral detection; dark-field illumination; diascopic configuration

\section{Introduction}

Flow cytometry is a powerful technique for the measurement and analysis of various characteristics of cell samples, and is used in applications ranging from immunophenotyping [1,2] to gene diagnosis [3,4], bacterial analysis [5], clinical hematology diagnosis [6-8], and even food industry applications [9]. Accordingly, various discrete polychromatic detection systems [10] and spectral analysis systems $[11,12]$ have been developed in recent decades. In previous studies, this was generally achieved by using multiple light sources, filter sets, and photon detectors [13-16]. Delicate and luxurious fluorescence illumination systems were adopted to produce multiple fluorescence detection signals with different colors [14]. The results showed that the developed optical systems permitted the analysis of up to 19 different parameters of quantum-dot-labeled samples. However, these approaches relied on large-scale optical components and automation processes to construct bench-top cytometry systems. Alternatively, some other researchers have proposed micro-scale cytometry chips incorporating buried optical fibers to couple and transmit the excitation light and to collect the fluorescence signals, respectively [5,17-21]. However, whilst such devices provide an effective means of accomplishing multi-parametric analyses, the requirement for delicate optical components and 
multiple optical filters and photo-detectors greatly increases their cost and complexity. Furthermore, the fabrication processes for these microfluidic chips are relatively delicate and time consuming.

In spectral detection schemes, a multi-color detection capability was achieved using a single photo detector (e.g., a charge-coupled device (CCD), camera, or photomultiplier tube (PMT)) equipped with a grating [22], integrated micro-spectrometer [23], bio-photonic detector [24], or tunable filter [11,25]. For example, Goddard et al. [26] utilized a diffraction grating to disperse the fluorescence signals emitted from the cells or microspheres passing through the interrogation region of the cytometer, and then used a CCD image sensor to analyze the spectral distribution of the collected signals. Individual fluorophores in multiple-tagged samples were successfully detected with high resolution. Typically, most spectral detection schemes use a single monochromatic light source, such as an argon laser with a UV wavelength $(488 \mathrm{~nm})$, to excite the fluorescent-labeled samples in order to broaden the fluorescence spectra and simplify the experimental setup. However, the use of a monochromatic excitation source limits the number of fluorescent probes which can be distinguished by the cytometry system. Several reports have indicated that this problem can be overcome by replacing the conventional organic fluorophores with quantum dot reagents due to their higher fluorescence emissions [27-29]. Similarly, Watson et al. [30] achieved a multi-parameter analysis capability by replacing the CCD detector in the cytometer by a Raman spectral detection system. Raman flow cytometry makes the multi-parameter, multiplexed measurement of cells and particles using a single detector and light source possible. However, such systems involve a more complicated sample-labeling procedure than those required for conventional organic fluorescent probes. In this regards, the practical applications of this method are hindered.

Dark-field illumination is generally used for the detection and tracking of small or transparent particles in a solution near the surface [31,32]. The use of dark-field illumination in accomplishing multi-color fluorescence detection on a single microfluidic chip for capillary electrophoresis applications was also successfully demonstrated [33,34]. A dark-field configuration was also used to quantify and characterize particles and cells in accordance with their light scattering properties [35]. The concept for developing a simple yet effective technique for the simultaneous detection of mixed samples comprising cells/particles with different sizes and with different fluorescence labels on a microfluidic chip without the need for spatial filters or delicate optical components has been reported [36]. In the present study, an objective-type dark-field condenser equipped with a white light source with continuous wavelengths $(400-900 \mathrm{~nm})$ is used to induce scattered light and fluorescence signals from particles or cells as they pass through the interrogation region of the microfluidic chip. The scattered light and fluorescence signals are then collected by an ultraviolet-visible-near infrared (UV-Vis-NIR) spectrometer. The resulting spectrogram is then processed using a simple linear superposition scheme in order to extract the fluorescence, absorbance, and side-scatter properties of the various particles or cells within the sample. This work focuses on the improved computation algorithm which significantly improves the ability for the developed system to discriminate more fluorescence dyes and real biological samples, simultaneously. The feasibility of the proposed multispectral cytometer is demonstrated via the detection and analysis of a dummy sample comprising polystyrene beads with green, red, and crimson fluorescence labels, respectively, and the dimensions of 5, 10 and $15 \mu \mathrm{m}$. The real biological applicability of the proposed configuration is then confirmed via the analysis of a bio-sample containing gastric epithelial (AGS) cells stained with two different dyes, namely Trypan-blue and Erythrosin-bluish.

\section{Materials and Methods}

\subsection{Working Principle}

Figure 1 illustrates the working principle of the proposed multi-wavelength detection applied to the micro-flow cytometer. As shown, a white light source with a continuous wavelength spectrum $(400-900 \mathrm{~nm})$ is passed through an objective lens equipped with a light-stop such that it forms a 
hollow-cone-shaped illumination region focused on the microfluidic channel. Significantly, the white light source provides an effective excitation light over a broad spectrum, and is therefore suitable for the excitation of many different fluorescence and absorbance dyes without the need for spatial filters. When particles labeled with fluorescent probes pass through the interrogation region of the cytometer, part of the incident light is absorbed and subsequently emitted as fluorescence, while part of the incident light is scattered. Thus, the spectrogram of the signal acquired at the spectrometer contains information relating to the absorbance, fluorescence, and scattered-light properties of the particles, respectively.

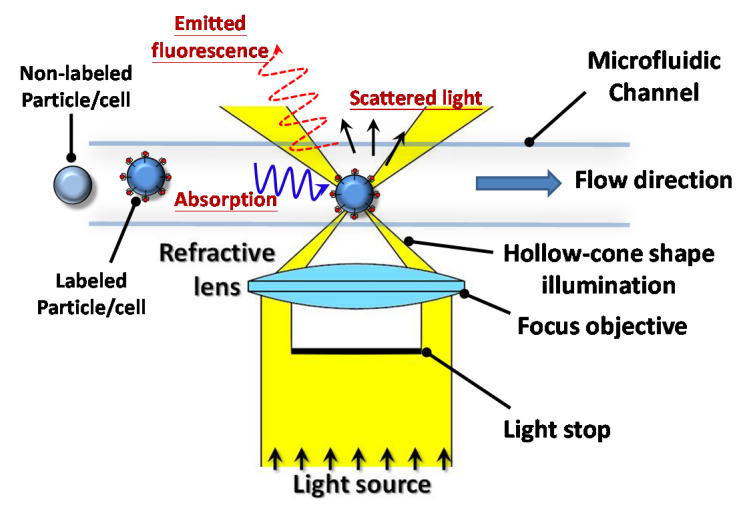

Figure 1. Working principle of the proposed diascopic illumination technique in multispectral cytometry analyses.

\subsection{Instrumental Setup and Flow Cytometry}

Figure 2 presents a schematic representation of the proposed multi-spectral micro-flow cytometry system. The bulk of the experimental system is built on an inverted microscope (Eclipse TE2000U, Nikon, Tokyo, Japan) equipped with a high numerical aperture (N.A.) objective lens $\left(\mathrm{CFI}_{60} 20 \times / 0.75\right.$, Nikon, Tokyo, Japan) and a light-stop film as the excited unit. In accordance with the proof-of-concept study performed in [36], the spatial resolution of the cytometer was further enhanced by passing the light emitted from the dark-field condenser through a pinhole prior to entering the high N.A. objective lens. Additionally, a small interrogation region is beneficial to particle/cell detection and counting, and the experiments were performed using a circular pinhole with a diameter of $1 \mathrm{~mm}$ for forming the interrogation region with a diameter of $100 \mu \mathrm{m}$.

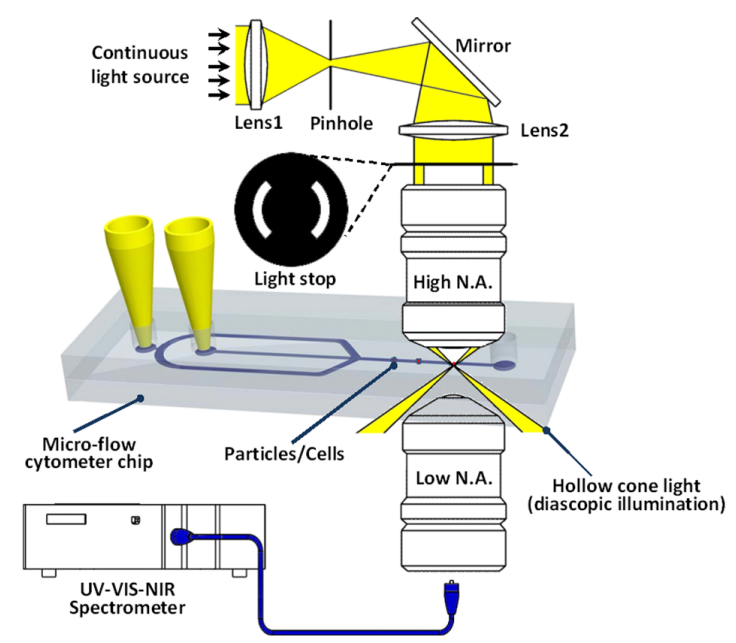

Figure 2. Schematic for the experimental setup of the proposed multi-parameter cytometry detection system. N.A., numerical aperture; UV-Vis-NIR, ultraviolet-visible light-near infrared. 
The light emitted from the interrogation region of the cytometer was collected by a low N.A. objective lens $\left(\mathrm{CFI}_{60} 20 \times / 0.45\right.$, Nikon, Tokyo, Japan) and was then coupled into a UV-Vis-NIR spectrometer (HR4000, Ocean Optics, Dunedin, FL, USA) via a multi-mode fused silica fiber (P400-2-UV-Vis, Ocean Optic, Dunedin, FL, USA). Note that an objective lens with a lower N.A. than that of the excitation objective lens was deliberately chosen on the detection side of the cytometer in order to restrict the collected light to just the scattered light and fluorescence signals emitted from the particles. The detection signal was processed by the spectrometer and then collected using a personal computer via a USB interface and software (OOIBase32, Ocean Optics, Dunedin, FL, USA) at a sampling rate of 20 acquisitions per second. Finally, the spectra were analyzed and post-processed by a proprietary program written in Matlab ${ }^{\circledR}$ (R2012, The MathWorks, Natick, MA, USA). In order to meet the optical detection scheme of this study, the microfluidic chip was fabricated in low-cost microscope slides with a rapid fabrication process [37]. The details regarding the chip design and the reagents for the experiments are provided in the supplementary information.

To confirm the focusing capability of the two sheath flows, an initial experiment was performed using Rhodamine B as the sample solution. Figure 3 presents the visualization results obtained using a general fluorescence microscope after the vacuum pump was removed, and the sheath and sample moved under the effects of inertia and hydrostatic forces alone. The results presented in Figure $3 a$ confirm that the sheath flows confine the sample flow to a narrow stream within the center of the microchannel. The width of the focused stream is dependent on the sample and sheath flow rates [38], and in the present case, was found to have a value of $20 \mu \mathrm{m}$. However, a slight diffusion of the focused stream was observed in the downstream region of the chip due to the low flow rate.

Having confirmed the focusing capability of the two sheath flows, a second experiment was performed using a sample containing red fluorescent-labeled particles with a size of $15 \mu \mathrm{m}$. The corresponding visualization results are presented in Figure $3 \mathrm{~b}$. It can be seen that the focusing effect causes the particles to flow through the interrogation region of the chip in a single-line fashion, thereby permitting their individual characterization and counting. The linear velocity of the focused sample stream was about $500 \mu \mathrm{m} / \mathrm{s}$ for the particle movement, and the velocity was necessary to provide sufficient exposure time for the spectral measurement using the UV-Vis-NIR spectrometer with an integrated time of $20 \mathrm{~ms}$.
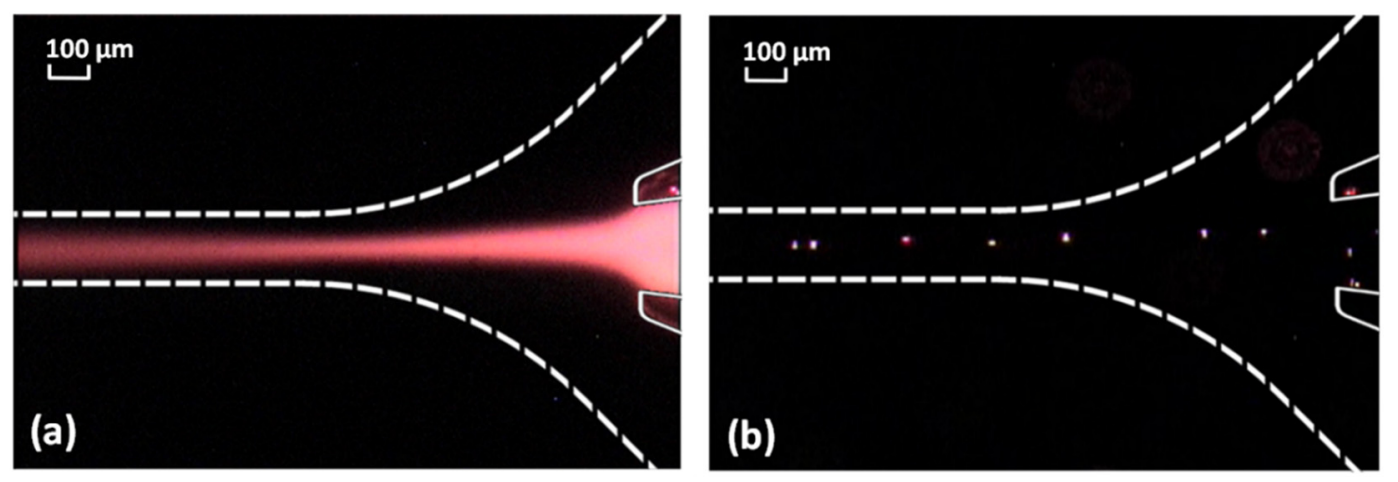

Figure 3. (a) Fluorescent dye squeezed into a narrow stream by neighboring sheath flows, (b) focused red fluorescent-labeled particles with a size of $15 \mu \mathrm{m}$ focused into a single stream for detection and analysis purposes.

\subsection{Reagents and Bio-Sample Preparation}

The feasibility of the proposed micro-flow cytometer was evaluated initially using blank polystyrene beads and red, green, and crimson fluorescent-labeled beads of various sizes. Other than the crimson polystyrene beads $(10 \mu \mathrm{m}, 625 / 345$, Invitrogen, Thermo Fisher Scientific Inc., Waltham, MA, USA), all of the beads were purchased from Thermo Scientific (Duke Standards) Co. in the USA. The real-world applicability of the proposed cytometer was then investigated using 
a bio-sample containing stained and non-stained AGS cells. In preparing the bio-sample, AGS cells in phosphate-buffered saline (PBS, Invitrogen, Waltham, MA, USA) were fixed with $70 \% \mathrm{EtOH}(v / v$, cell:EtOH $=1: 5$, J.T. Baker, Center Valley, PA, USA) at $-20{ }^{\circ} \mathrm{C}$ overnight. The fixed cells were washed by centrifugation and re-suspended in $0.5 \mathrm{~mL}$ PBS buffer before adding $0.5 \mathrm{~mL}$ high salt concentration buffer $\left(0.2 \mathrm{M} \mathrm{Na}_{2} \mathrm{HPO}_{4}\right.$ and $0.1 \mathrm{M}$ citric acid, Sigma-Aldrich, St. Louis, MO, USA) for 5 min. The cells were then collected by centrifugation and re-suspended in a $0.5 \mathrm{~mL}$ buffer solution mixed with either $20 \mu \mathrm{L}$ Trypan-blue (4\% $w / v$, Invitrogen, Waltham, MA, USA) or $20 \mu \mathrm{L}$ Erythrosin-bluish $(4 \% w / v$, Sigma-Aldrich, St. Louis, MO, USA) staining solution for $10 \mathrm{~min}$.

\section{Results and Discussion}

\subsection{Spectral Analysis and Performance Measurement}

The discrimination capability of the micro-flow cytometer was evaluated using a mixed sample comprising green fluorescent-labeled beads $(468 / 508 \mathrm{~nm})$, red fluorescent-labeled beads $(542 / 612 \mathrm{~nm})$, crimson fluorescent-labeled beads $(625 / 645 \mathrm{~nm})$, and non-labeled beads (blank) with diameters of 5 , 15,10 and $12 \mu \mathrm{m}$, respectively. Figure 4a presents a dark-field image of the particles captured through the eyepiece lens of the microscope using a digital camera (SP-350, Olympus, Center Valley, PA, USA) with an exposure time of $25 \mathrm{~ms}$. The image clearly shows a scattering phenomenon with different color expression at the boundary of each polystyrene bead due to the difference in refractive indices of the particles (1.59 at $589 \mathrm{~nm}$ ) and the sample solution (deionized water, 1.33 at $589 \mathrm{~nm}$ ), respectively. It is noted that the scatter patterns correspond to the pattern of the light-stop film. Figure $4 \mathrm{~b}$ presents the detected wavelength spectra of the labeled and non-labeled particles. The difference in the four spectra enables the different particles to be reliably identified. Specifically, the green, red, and crimson fluorescent-labeled particles result in a scattering of the green, red, and blue light, respectively. By contrast, the non-labeled particles simply reflect the white excitation light, i.e., the normalized detected spectrum is identical to the excitation spectrum. It is observed that the spectra of the green and red fluorescent-labeled particles exhibit slighter absorption at the shorter wavelengths than the emitted florescence corresponding to the excitation wavelength of each fluorescence label. However, in general the emission spectra of the green and red fluorescence labels dominate the overall color expression of the particles in the dark-field image. Therefore, the color expressions of the green- and red-labeled particles correspond to the detected spectra (i.e., green fluorescence at $502 \mathrm{~nm}$ and red fluorescence at $580 \mathrm{~nm}$ ). By contrast, the spectrum of the crimson fluorescent-labeled particles exhibits strong and broad band absorption at wavelengths between 550 and $650 \mathrm{~nm}$ and has a fluorescence peak at $690 \mathrm{~nm}$.
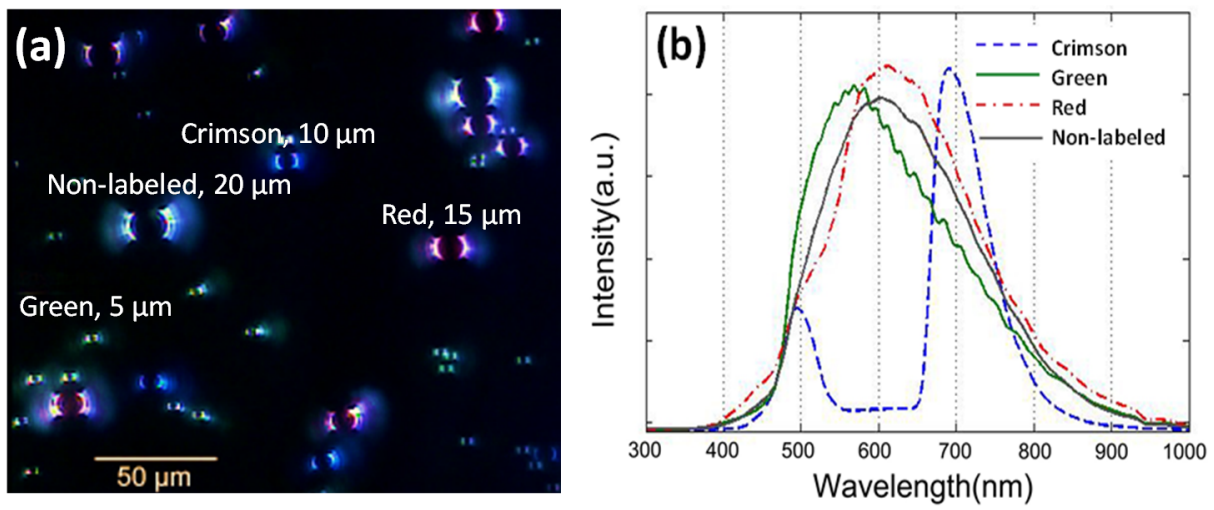

Figure 4. (a) Images obtained under diascopic illumination of green-labeled ( $5 \mu \mathrm{m})$, red-labeled $(15 \mu \mathrm{m})$, crimson-labeled $(10 \mu \mathrm{m})$, and non-labeled $(12 \mu \mathrm{m})$ polystyrene beads. (b) Corresponding detected wavelength spectra. Note that the spectrum for the non-labeled particle in $(\mathbf{b})$ was used as reference spectra and was used to calculate the fluorescent, absorbance, and side-scatter indices. 
Although the detected spectra provide the means to distinguish between the different fluorescent-labeled particles, real-world cytometry applications require a more quantitative analysis of the sample. Accordingly, the sampled spectrogram data acquired in the previous experiments were processed using a simple linear superposition scheme in order to extract the corresponding absorption, fluorescence, and side-scatter information. In general, the detected spectrum, $I_{d}(\lambda)$, (i.e., the lines for non-labeled particle in Figure $4 \mathrm{~b}$ ) composed of absorption and fluorescence spectral signals can be expressed as

$$
I_{\mathrm{d}}(\lambda)=I_{\mathrm{abs}}(\lambda)+I_{\text {fluor }}(\lambda)+I_{\mathrm{ss}}(\lambda)
$$

where $I_{\mathrm{abs}}(\lambda)$ and $I_{\text {fluor }}(\lambda)$ are the absorbance (negative sign) and fluorescence (positive sign) spectra of the specific fluorophore probe, respectively, $I_{\mathrm{sS}}(\lambda)$ is the side-scatter (positive sign) spectrum (i.e., the line for the labeled particle shown in Figure $4 \mathrm{~b}$ which is identical to the spectrum of non-labeled particles for the reference of the linear superposition calculation), and $\lambda$ is the wavelength.

The detection performance of the proposed system was further evaluated using a mixed sample comprising red fluorescent-labeled particles with dimensions of 3,7 and $15 \mu \mathrm{m}$, respectively. Figure $5 \mathrm{a}, \mathrm{b}$ present one-dimensional histograms showing the distribution of the particles within the sample in terms of their fluorescent indices and side-scatter indices, respectively. Note that the fluorescent index $\left(F l_{\text {index }}\right)$ is calculated by integrating the function of $I_{\text {fluor }}(\lambda)$ and provides a reliable index with which to compare the fluorescence levels of the different-sized particles. A similar method is used to calculate the absorbance $\left(A b s_{\text {index }}\right)$ and side-scatter $\left(S S_{\text {index }}\right)$ indices. In other words, the fluorescent, absorbance, and side-scatter indices are given by

$$
\begin{gathered}
F l_{\text {index }}=\int I_{\text {fluor }}(\lambda) d x \\
A b s_{\text {index }}=\int I_{\text {abs }}(\lambda) d x \\
S S_{\text {index }}=\int I_{\text {ss }}(\lambda) d x
\end{gathered}
$$
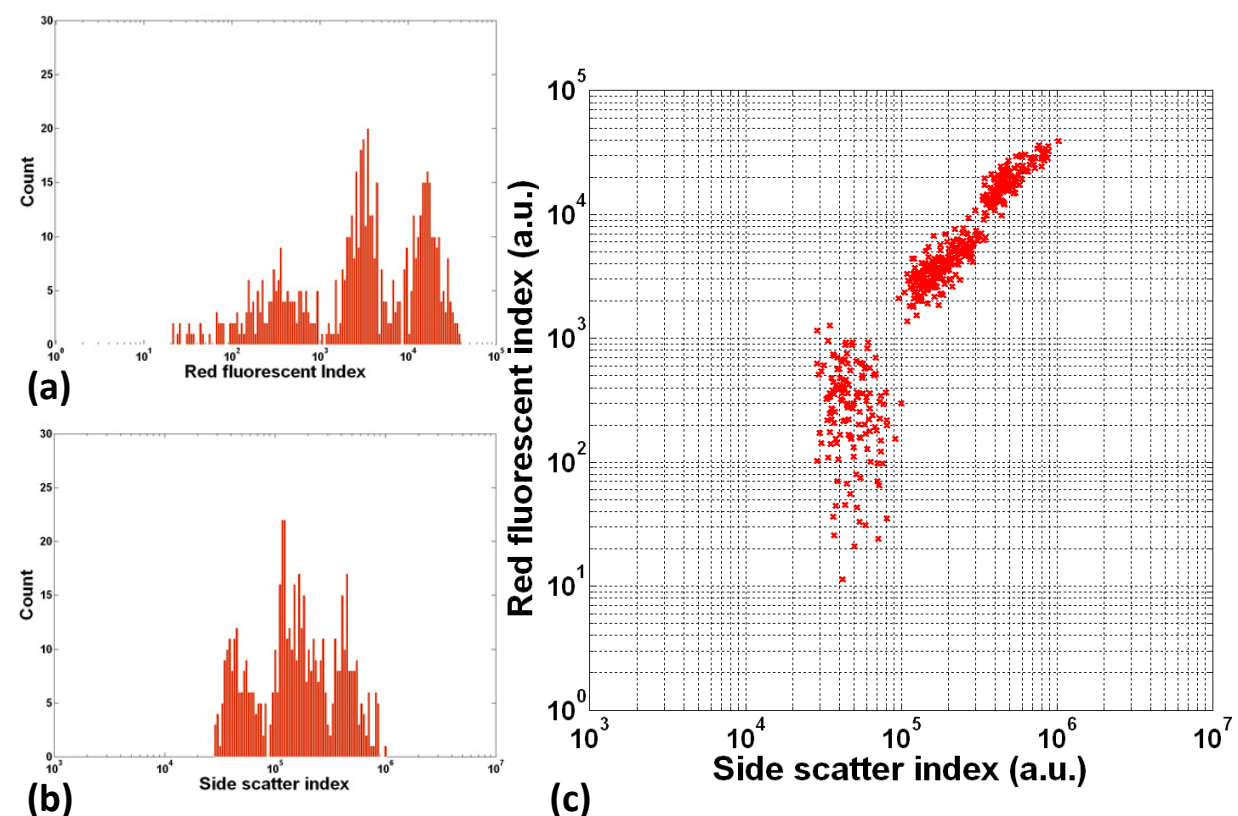

(c)

Figure 5. Flow cytometer detection results for mixed sample composed of particles with sizes of 3, 10 and $15 \mu \mathrm{m}$, respectively: (a) one-dimensional histogram for fluorescence emission, (b) one-dimensional histogram for side scatter, (c) scatter plot of fluorescent index and side-scatter index. 
In this application, the variations of fluorescence and side-scatter indices are both involved with the vertical position in the microfluidic channel due to a very shallow excitation region of the objective-type dark-field condenser. Although the diascopic configuration is an easy way to realize the advanced objective-type dark-field system for excitation of particles, it also brings the disadvantage for the quantification of fluorescence and side-scatter. When the fluorescence-labeled particles are higher or lower than the focal plane, fluorescence has a more obvious variation than side-scatter because of different excitation planes for fluorescence and side-scatter detection. An inspection of the fluorescence histogram presented in Figure 5a shows that the coefficients of variation (CV) for the populations of 3-, 7- and $15-\mu \mathrm{m}$ particles are $70.94 \%, 43.91 \%$ and $35.55 \%$, respectively. According to the side-scatter histogram (Figure $5 b$ ), the CVs of the three populations are $26.97 \%, 37.59 \%$ and $32.29 \%$, respectively. It is noted that these values are significantly lower than those in Figure 5a. Since the intensity for the scattered light was determined by the scattering surface of the passing particle, the bigger particle scattered more light such that particles with different sizes could be discriminated by the developed system. Figure $5 \mathrm{c}$ presents a scatter chart of the fluorescent indices and side-scatter indices of the particles within the mixed sample. The results confirm the ability of the proposed system to discriminate between particles of different sizes within the sample.

\subsection{Discrimination for Particles and Bio-Cells}

The ability of the developed cytometer to discriminate and count microspheres of different types and sizes was evaluated using a mixed sample comprised of green fluorescent-labeled particles $(7 \mu \mathrm{m})$, red fluorescent-labeled particles $(15 \mu \mathrm{m})$, crimson fluorescent-labeled particles $(10 \mu \mathrm{m})$, and non-labeled particles $(12 \mu \mathrm{m})$. Figure 6a presents the results obtained for the four sub-population clusters within the sample when classifying the particles in terms of the green, red, and crimson fluorescent indices. For clarity, Figure $6 \mathrm{~b}-\mathrm{d}$ present the 2D scatter plots obtained by plotting the three fluorescent indices against the side-scatter index. In general, the results confirm the ability of the proposed cytometer to simultaneously detect and count particles with different fluorescence-labels and sizes using a single white light source and no filter sets.
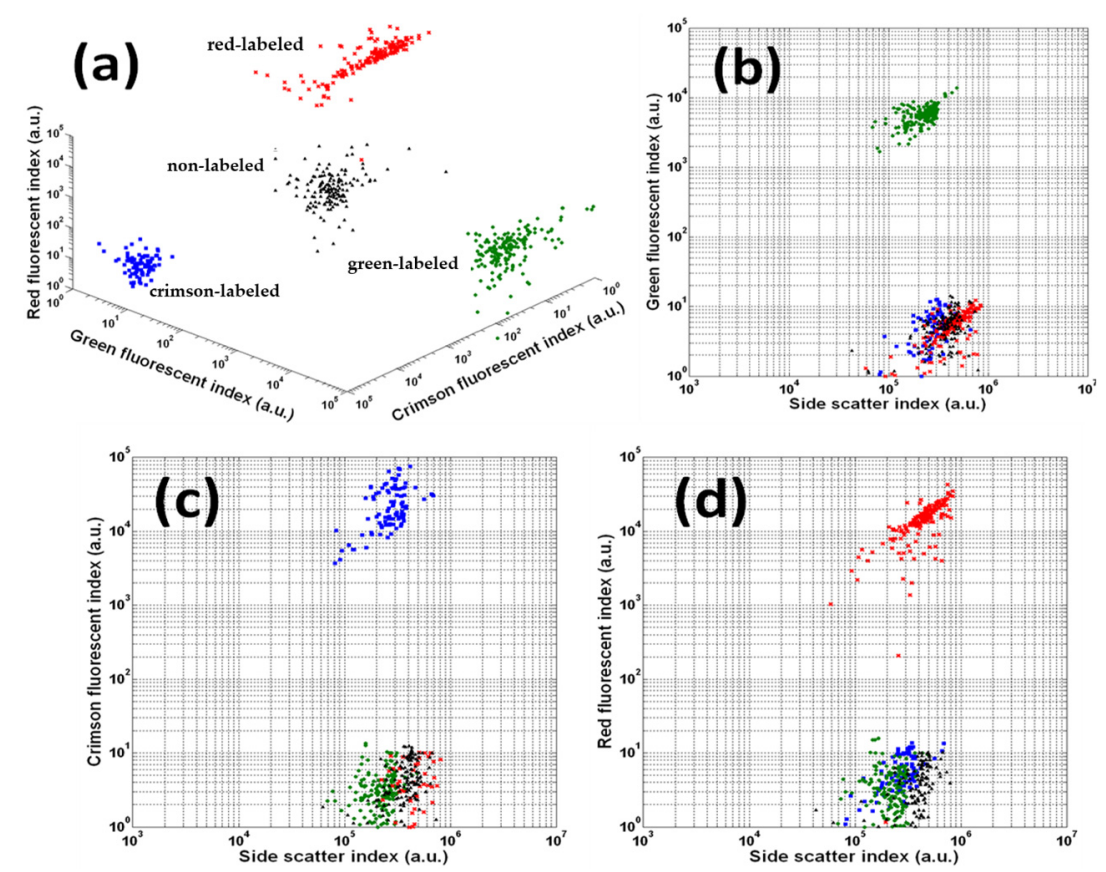

Figure 6. Flow cytometer detection results for the mixed sample composed of green-, red-, crimson-labeled and non-labeled particles: (a) 3D scatter plot without side-scatter index; (b-d) 2D scatter plots with different fluorescent and side-scatter indices. 
The feasibility of the proposed cytometer for practical bio-detection applications was evaluated using a sample comprising a mixture of Trypan-blue and Erythrosin-bluish stained AGS cells and unstained AGS cells. Figure 7 presents the dark-field images and detected spectra of the different cells. The dark-field images confirm the ability of the proposed illumination system to provide an effective excitation source for cells stained with different dyes. For microsphere samples, scattering occurs only at the boundary. However, cells and bio-samples contain both water and cytoplasma with a refractive index close to that of the buffer solution. As a result, the scattering phenomenon occurs not only at the boundary of the cells or bio-samples, but also at various points on their surface. Consequently, the dark-field images shown in Figure 7 exhibit a homogenous scattered color expression. When the cells are illuminated by the white light excitation source, certain wavelengths are absorbed (depending on the absorbance properties of the particular dye), whilst others are reflected or scattered. The Erythrosin-bluish stained cells absorb blue-green light $(400-550 \mathrm{~nm})$, and therefore have a red color when illuminated by the excitation light (see insets of Figure 7a). Similarly, the Trypan-blue-blue stained cells absorb green-red light (550-700 $\mathrm{nm})$, and therefore have a blue appearance. The non-labeled cell yields a peak intensity at a wavelength of around $510 \mathrm{~nm}$ due to the absorption of water at the near infrared range. As a result, it has a light green color.
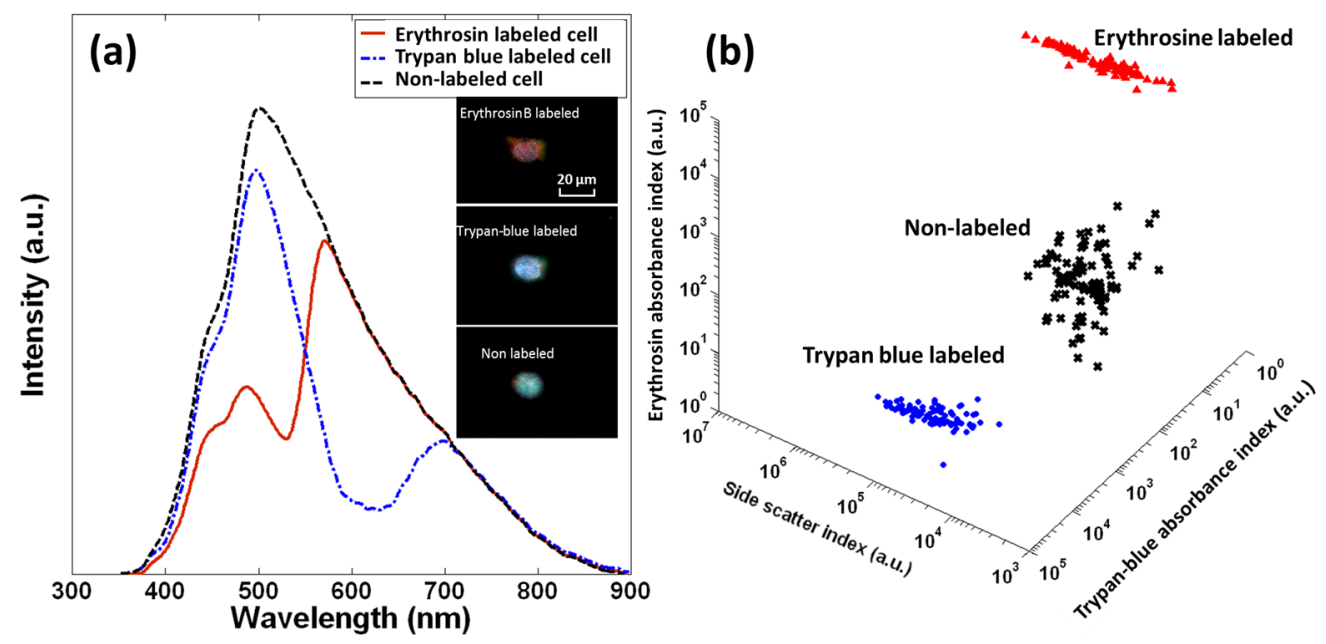

Figure 7. (a) Wavelength spectra of stained and non-stained gastric epithelial (AGS) cells. Note that the insets show the captured dark-field images of stained and non-stained AGS cells, respectively; (b) 3D scatter plot of the side-scatter index and the absorbance indices of stained AGS cells, respectively.

Figure $7 \mathrm{~b}$ presents the 3D scatter plots of the mixed sample shown in Figure 7a. The results confirm that the information-rich spectra obtained using the proposed dark-field illumination configuration enable the multi-parameter analysis and characterization of bio-samples containing cells or particles with different dimensions, optical properties, and dye labels (i.e., fluorescence or absorbance). As a result, the system provides a simple and low-cost alternative to conventional multi-color cytometer systems based on multiple laser illumination sources and filter sets.

\section{Conclusions}

This paper has presented a diascopic illumination configuration for the characterization and counting of cells and particles using a micro-flow cytometer chip. In the proposed approach, the cells and particles are illuminated by a white light source under dark-field conditions, and the corresponding detection spectra are acquired by a UV-Vis-NIR spectrometer. The spectrum data are then processed by a simple linear superposition scheme in order to extract the corresponding absorption, fluorescence, and side-scatter information. The extracted information is then analyzed using an improved computation model to characterize the sample components in terms of their size, number, and fluorescent labels. With the newly developed computation method, the developed 
configuration successfully discriminated between both micro-particles with four different fluorescence labels, and a mixed bio-sample comprising Trypan-blue and Erythrosin-bluish stained AGS cells. The results have confirmed that the spectra obtained using the dark-field illumination configuration enable multi-parameter analysis and characterization of samples with different sizes, optical properties, and fluorescent labels. Significantly, the proposed configuration utilizes a single white light source and has no need for spatial filters or delicate optical components. Consequently, it provides an effective, low-cost solution for the multi-color detection, identification, and classification of bio-samples within a single microchannel.

Acknowledgments: We acknowledge the financial support from the Ministry of Science and Technology (MOST) of Taiwan, which was greatly appreciated.

Author Contributions: Shi-Wei Lin and Che-Hsin Lin both conceived and designed the experiments; Shi-Wei Lin performed the experiments and analyzed the data; Shi-Wei Lin wrote the manuscript and Che-Hsin Lin conducted it into paper.

Conflicts of Interest: The authors declare no conflict of interest.

\section{References}

1. Cheng, M.Y.; Lin, C.L.; Lai, Y.T.; Yang, Y.J. A polymer-based capacitive sensing array for normal and shear force measurement. Sensors 2010, 10, 10211-10225. [CrossRef] [PubMed]

2. Roederer, M.; Brenchley, J.M.; Betts, M.R.; De Rosa, S.C. Flow cytometric analysis of vaccine responses: How many colors are enough? Clin. Immunol. 2004, 110, 199-205. [CrossRef] [PubMed]

3. Zhang, H.Y.; Sun, H. Up-regulation of foxp3 inhibits cell proliferation, migration and invasion in epithelial ovarian cancer. Cancer Lett. 2010, 287, 91-97. [CrossRef] [PubMed]

4. Lee, J.; Kim, I.S.; Yu, H.W. Flow cytometric detection of bacillus spoOA gene in biofilm using quantum dot labeling. Anal. Chem. 2010, 82, 2836-2843. [CrossRef] [PubMed]

5. Kim, J.S.; Anderson, G.P.; Erickson, J.S.; Golden, J.P.; Nasir, M.; Ligler, F.S. Multiplexed detection of bacteria and toxins using a microflow cytometer. Anal. Chem. 2009, 81, 5426-5432. [CrossRef] [PubMed]

6. Zheng, S.Y.; Nandra, M.S.; Shih, C.Y.; Li, W.; Tai, Y.C. Resonance impedance sensing of human blood cells. Sens. Actuators A 2008, 145, 29-36. [CrossRef]

7. Zheng, S.Y.; Lin, J.C.H.; Kasdan, H.L.; Tai, Y.C. Fluorescent labeling, sensing, and differentiation of leukocytes from undiluted whole blood samples. Sens. Actuators B 2008, 132, 558-567. [CrossRef]

8. Orlova, D.Y.; Yurkin, M.A.; Hoekstra, A.G.; Maltsev, V.P. Light scattering by neutrophils: Model, simulation, and experiment. J. Biomed. Opt. 2008. [CrossRef] [PubMed]

9. Garcia-Cordero, J.L.; Barrett, L.M.; O'Kennedy, R.; Ricco, A.J. Microfluidic sedimentation cytometer for milk quality and bovine mastitis monitoring. Biomed. Microdevices 2010, 12, 1051-1059. [CrossRef] [PubMed]

10. Darzynkiewicz, Z.; Bedner, E.; Li, X.; Gorczyca, W.; Melamed, M.R. Laser-scanning cytometry: A new instrumentation with many applications. Exp. Cell Res. 1999, 249, 1-12. [CrossRef] [PubMed]

11. Mathur, A.; Kelso, D.M. Multispectral image analysis of binary encoded microspheres for highly multiplexed suspension arrays. Cytom. Part A 2010, 77A, 356-365. [CrossRef] [PubMed]

12. Barat, D.; Spencer, D.; Benazzi, G.; Mowlem, M.C.; Morgan, H. Simultaneous high speed optical and impedance analysis of single particles with a microfluidic cytometer. Lab Chip 2012, 12, 118-126. [CrossRef] [PubMed]

13. Bigos, A.; Baumgarth, N.; Jager, G.C.; Herman, O.C.; Nozaki, T.; Stovel, R.T.; Parks, D.R.; Herzenberg, L.A. Nine color eleven parameter immunophenotyping using three laser flow cytometry. Cytom. Part A 1999, 36, 36-45. [CrossRef]

14. Perfetto, S.P.; Chattopadhyay, P.K.; Roederer, M. Innovation-seventeen-colour flow cytometry: Unravelling the immune system. Nat. Rev. Immunol. 2004, 4, 648-655. [CrossRef] [PubMed]

15. Lin, C.H.; Lee, G.B.; Chen, S.H.; Chang, G.L. Micro capillary electrophoresis chips integrated with buried su-8/sog optical waveguides for bio-analytical applications. Sens. Actuators A 2003, 107, 125-131. [CrossRef]

16. Guo, J.H.; Huang, X.W.; Shi, D.Y.; Yu, H.; Ai, Y.; Li, C.M.; Kang, Y.J. Portable resistive pulse-activated lens-free cell imaging system. Rsc Adv. 2014, 4, 56342-56345. [CrossRef]

17. Golden, J.P.; Kim, J.S.; Erickson, J.S.; Hilliard, L.R.; Howell, P.B.; Anderson, G.P.; Nasir, M.; Ligler, F.S. Multi-wavelength microflow cytometer using groove-generated sheath flow. Lab Chip 2009, 9, 1942-1950. [CrossRef] [PubMed] 
18. Lin, C.H.; Lee, G.B. Micromachined flow cytometers with embedded etched optic fibers for optical detection. J. Micromech. Microeng. 2003, 13, 447-453. [CrossRef]

19. Tung, Y.C.; Zhang, M.; Lin, C.T.; Kurabayashi, K.; Skerlos, S.J. Pdms-based opto-fluidic micro flow cytometer with two-color, multi-angle fluorescence detection capability using pin photodiodes. Sens. Actuators B 2004, 98, 356-367. [CrossRef]

20. Kennedy, M.J.; Stelick, S.J.; Sayam, L.G.; Yen, A.; Erickson, D.; Batt, C.A. Hydrodynamic optical alignment for microflow cytometry. Lab Chip 2011, 11, 1138-1143. [CrossRef] [PubMed]

21. Schonbrun, E.; Gorthi, S.S.; Schaak, D. Microfabricated multiple field of view imaging flow cytometry. Lab Chip 2012, 12, 268-273. [CrossRef] [PubMed]

22. Isailovic, D.; Li, H.W.; Phillips, G.J.; Yeung, E.S. High-throughput single-cell fluorescence spectroscopy. Appl. Spectrosc. 2005, 59, 221-226. [CrossRef] [PubMed]

23. Hu, Z.X.; Glidle, A.; Ironside, C.; Cooper, J.M.; Yin, H.B. An integrated microspectrometer for localised multiplexing measurements. Lab Chip 2015, 15, 283-289. [CrossRef] [PubMed]

24. Chandrasekaran, A.; Packirisamy, M. Integrated microfluidic biophotonic chip for laser induced fluorescence detection. Biomed. Microdevices 2010, 12, 923-933. [CrossRef] [PubMed]

25. Sunamura, M.; Maruyama, A.; Tsuji, T.; Kurane, R. Spectral imaging detection and counting of microbial cells in marine sediment. J. Microbiol. Methods 2003, 53, 57-65. [CrossRef]

26. Goddard, G.; Martin, J.C.; Naivar, M.; Goodwin, P.M.; Graves, S.W.; Habbersett, R.; Nolan, J.P.; Jett, J.H. Single particle high resolution spectral analysis flow cytometry. Cytom. Part A 2006, 69A, 842-851. [CrossRef] [PubMed]

27. Ferrari, B.C.; Bergquist, P.L. Quantum dots as alternatives to organic fluorophores for cryptosporidium detection using conventional flow cytometry and specific monoclonal antibodies: Lessons learned. Cytom. Part A 2007, 71A, 265-271. [CrossRef] [PubMed]

28. Chattopadhyay, P.K.; Price, D.A.; Harper, T.F.; Betts, M.R.; Yu, J.; Gostick, E.; Perfetto, S.P.; Goepfert, P.; Koup, R.A.; De Rosa, S.C.; et al. Quantum dot semiconductor nanocrystals for immunophenotyping by polychromatic flow cytometry. Nat. Med. 2006, 12, 972-977. [CrossRef] [PubMed]

29. Yun, H.; Bang, H.; Min, J.; Chung, C.; Chang, J.K.; Han, D.C. Simultaneous counting of two subsets of leukocytes using fluorescent silica nanoparticles in a sheathless microchip flow cytometer. Lab Chip 2010, 10, 3243-3254. [CrossRef] [PubMed]

30. Watson, D.A.; Brown, L.O.; Gaskill, D.R.; Naivar, M.; Graves, S.W.; Doorn, S.K.; Nolan, J.P. A flow cytometer for the measurement of raman spectra. Cytom. Part A 2008, 73A, 119-128. [CrossRef] [PubMed]

31. Braslavsky, I.; Amit, R.; Ali, B.M.J.; Gileadi, O.; Oppenheim, A.; Stavans, J. Objective-type dark-field illumination for scattering from microbeads. Appl. Opt. 2001, 40, 5650-5657. [CrossRef] [PubMed]

32. Piper, T.; Piper, J. Variable phase dark-field contrast-A variant illumination technique for improved visualizations of transparent specimens. Microsc. Microanal. 2012, 18, 343-352. [CrossRef] [PubMed]

33. Lin, S.W.; Hsu, J.H.; Chang, C.H.; Lin, C.H. Objective-type dark-field system applied to multi-wavelength capillary electrophoresis for fluorescent detection and analysis. Biosens. Bioelectron. 2009, 25, 450-455. [CrossRef] [PubMed]

34. Lin, S.W.; Chang, G.L.; Lin, C.H. Novel wave length-resolved fluorescence detection for a high-throughput capillary electrophoresis system under a diascopic configuration. J Chromatogr. A 2008, 1192, 198-201. [CrossRef] [PubMed]

35. Rebner, K.; Schmitz, M.; Boldrini, B.; Kienle, A.; Oelkrug, D.; Kessler, R.W. Dark-field scattering microscopy for spectral characterization of polystyrene aggregates. Opt. Express 2010, 18, 3116-3127. [CrossRef] [PubMed]

36. Lin, S.W.; Wang, P.Y.; Chen, A.; Chang, C.H.; Lin, C.H. Wavelength-resolved flow cytometer under a dark-field illumination configuration. IEEE Sens. J. 2011, 11, 2845-2851. [CrossRef]

37. Lin, C.H.; Lee, G.B.; Lin, Y.H.; Chang, G.L. A fast prototyping process for fabrication of microfluidic systems on soda-lime glass. J. Micromech. Microeng. 2001, 11, 726-732. [CrossRef]

38. Lee, G.B.; Lin, C.H.; Chang, G.L. Micro flow cytometers with buried su-8/sog optical waveguides. Sens. Actuators A 2003, 103, 165-170. [CrossRef]

(C) 2016 by the authors; licensee MDPI, Basel, Switzerland. This article is an open access article distributed under the terms and conditions of the Creative Commons Attribution (CC-BY) license (http://creativecommons.org/licenses/by/4.0/). 